\title{
Radiocarbon
}

1972

\section{ALGIERS RADIOCARBON MEASUREMENTS II}

O. RAHMOUNI, C. ROUSSILLOT, and F. ARMANET

Service des Applications Nucleaires, Algiers, B.P. 1147, Algeria

The following series of dates contains most of the measurements obtained since our last list. Procedures of measurements and calculation are as previously described (R., 1970, v. 12, p. 353-357).

\section{ACKNOWLEDGMENTS}

We are grateful to S. A. Boutemine and N. Abdelmoumen for their help in routine work of chemical preparation of samples, and we thank A. Drizi for his assistance in electronics. Sample descriptions were prepared in collaboration with submitters and collectors of samples. Most of them were submitted by the Centre Algerien de Recherches Archéologiques, Préhistoriques et Ethnographiques (C.A.R.A.P.E.), the others were submitted by the Service des Antiquités d'Algérie.

\section{Crique des Pêcheurs series, Algeria}

\section{SAMPLE DESGRIPTIONS}

Various shells in archaeologic layer enclosed in sand hills at Bou Aïchem, Kristel, Willaya Oran ( $35^{\circ} 48^{\prime} \mathrm{N}$ Lat, $0^{\circ} 29^{\prime} \mathrm{W}$ Long), Algeria. Coll. 1968 and subm. by F. E. Roubet, C.A.R.A.P.E.

ALG-25. Crique des Pêcheurs 136

$10,215 \pm 400$ Various shells, remains of food.

ALG-26. Crique des Pêcheurs 140 8265 B.C.

Ostrich egg shells.

General Comment: controversy over industry; some authors consider these layers Neolithic culture. Previously estimated Epipaleolithic age was confirmed (Roubet, 1947).

\section{ALG-27. Sidi Tosni-Columnata}

$10,800 \pm 425$

$\mathbf{8 8 5 0}$ B.C.

Soft water shells from Columnata, Sidi Hosni, Willaya Tiaret $\left(35^{\circ}\right.$ $27^{\prime} 30^{\prime \prime}$ E Long, $1^{\circ} 31^{\prime} 41^{\prime \prime} \mathrm{N}$ Lat), Algeria. Coll. 1969 and subm. by C. Brahimi, C.A.R.A.P.E. Comment: layer presents a lateral and vertical stratigraphy for Epipaleolithic and Neolithic culture. Ibero-Maurusian culture never dated in this area (Brahimi, 1970).

\section{ALG-28. El Haouita 1}

$8220 \pm 820$

6270 B.C.

Charcoal from embankment at El Haouita, in Saharian Atlas, Willaya Laghouat $\left(37^{\circ} 41^{\prime} \mathrm{N}\right.$ Lat, $0^{\circ} 11^{\prime} \mathrm{E}$ Long), Algeria. Coll. 1968 and subm. by G. Aumassip, C.A.R.A.P.E. Comment: expected age of em- 
bankment: Würm period; it could have dated a new industry. Error was caused by mixing of the gas from the sample with a gas of known activity because of the small quantity of charcoal (Aumassip, 1970).

\section{ALG-29. Guentis 146}

\section{$\mathbf{5 2 5 0}$ B.C.}

Terrestrial shells (Helix) from upper level, $50 \mathrm{~cm}$, in a snailery at Guentis, Nemenchas country ( $30^{\circ} 0^{\prime} \mathrm{N}$ Lat, $7^{\circ} 18^{\prime} 15^{\prime \prime} \mathrm{E}$ Long), Algeria. Coll. 1968 and subm. by Roubet. Comment: in this layer were found implements comparable to Typical Capsian. This Epipaleolithic culture extending from Gafsa, Tunisia to Tebessa, Algeria had already been dated in Tunisia. First age value in Algeria (Roubet, 1968).

ALG-31. Djorf Torba

\section{A.D. 1870}

$80 \pm 110$

Charcoal from Djorf Torba, Béchar, Willaya Saoura $\left(31^{\circ} 30^{\prime} \mathrm{N}\right.$ Lat, $2^{\circ} 10^{\prime} \mathrm{W}$ Long), Algeria. Coll. 1967 by H. Lhote and subm. by G. Camps, C.A.R.A.P.E. Comment: this sample could have dated a pre-Islamic funeral monument. Unexpected age remains difficult to explain.

\section{ALG-32. Assech 3}

$5300 \pm 230$

Charcoal from Assech, Oued Mya, Willaya Oasis $\left(31^{\circ} 0^{\prime} \mathrm{N}\right.$ Lat, $4^{\circ} 40^{\prime}$ E Long), Algeria. Coll. 1969 and subm. by G. Aumassip. Comment: Neolithic culture (Aumassip, 1970).

\section{ALG-33. Botma Si Mamar}

$6600 \pm 250$

Terrestrial shells (Helix) in a deep level $(80$ to $100 \mathrm{~cm})$ from Botma Si Mamar, Sidi Khaled, Willaya Batna ( $34^{\circ} 22^{\prime}$ N Lat, $4^{\circ} 53^{\prime}$ E Long), Algeria. Coll. 1967 and subm. by D. Grébenart, C.A.R.A.P.E. Comment: Neolithic culture without pottery (Grébenart, 1970).

\section{ALG-34. Les Deux Oeufs}

$5500 \pm 125$

3350 B.C.

Ostrich egg shells in deepest level of layer, at Les Deux Oeufs, Ouargla, Oasis area ( $31^{\circ} 58^{\prime} \mathrm{N}$ Lat, $5^{\circ} 8^{\prime} \mathrm{E}$ Long), Algeria. Coll. 1966 and subm. by G. Aumassip. Comment: Neolithic culture (Aumassip, 1970).

\section{ALG-35. Les Deux Mamelles}

$5550 \pm 225$

Various shells from Les Deux Mamelles near Mostaganem, Willaya Oran (36 $36^{\prime} 12^{\prime \prime} \mathrm{N}$ Lat, $0^{\circ} 10^{\prime} 32^{\prime \prime} \mathrm{E}$ Long), Algeria. Coll. 1968 and subm. by F. E. Roubet. Comment: purpose was to date a particular aspect of Neolithic layers on sea-side of Oran Country (Roubet, 1947).

\section{ALG-36. Tin Hinan 3}

$1480 \pm 130$

Wood from building material of monument at Abalessa, Taman- 
rasset, Oasis area (23 $\mathrm{N}$ Lat, $5^{\circ} 0^{\prime} \mathrm{E}$ Long), Algeria. Coll. 1930 and subm. by M. Mammeri and G. Camps. Comment: date of a protohistoric funeral monument.

\section{Hafir series, Algeria}

Charcoal from Hafir, Terni, Willaya Tlemcen $\left(34^{\circ} 45^{\prime} \mathrm{N}\right.$ Lat, $3^{\circ}$ 40' W Long), Algeria. Coll. 1968 and subm. by M. Couvert, C.A.R.A.P.E.

\section{ALG-38. Hafir 6}

$7530 \pm 170$

Charcoal.

ALG-49. Hafir 7

$6680 \pm 175$

Charcoal.

4730 в.C.

General Comment: scarce lithic, bone industry, and pottery mingled with plentiful charcoal from 7 varieties of trees. Their analysis proved past existence of species now extinct in area, such as Cedrus atlanticus and Pinus nigra (Couvert, 1970).

\section{ALG-39. Bou Zabaouine 168}

$4375 \pm 145$ 2425 B.C.

Charcoal from shelter in rocks at Bou Zabaouine Aïn M'lila, Willaya Constantine (36 1' $^{\prime} \mathrm{N}$ Lat, $6^{\circ} 3^{\prime} 2^{\prime \prime}$ E Long), Algeria. Coll. 1967 and subm. by C. Roubet. Comment: Neolithic of Capsian tradition. Excavation, teeming with arrow points, corroborates that of $\mathrm{B}$. Dedieu (Dedieu, 1965; Roubet, 1968).

\section{ALG-40. Kristel Jardins}

$7760 \pm 190$ $\mathbf{5 8 1 0}$ в.с.

Sea shells, remains of food, from Sta. des Tavertins, Willaya Oran (35 $48^{\prime} 24^{\prime \prime} \mathrm{N}$ Lat, $0^{\circ}$ 29' W Long), Algeria. Coll. 1969 and subm. by F. E. Roubet. Comment: this archaeologic layer, presently mostly cultivated, is close to Criques des Pêcheurs (ALG-25); thus, date is interesting for comparisons and proves the real existence of an ancient Neolithic culture in this country (Roubet, 1947).

\section{ALG-41. Tipasa 2}

$2630 \pm 105$

680 B.c.

Snail shells from Matares, Tipasa, Willaya Algiers $\left(36^{\circ} 30^{\prime} \mathrm{N}\right.$ Lat, $2^{\circ} 26^{\prime}$ E Long), Algeria. Coll. 1969 and subm. by M. Bouchnaki, Service des Antiquités, Alger. Comment: sample from sand-hill near Roman tomb. Age older than expected.

\section{ALG-42. El Hadjar Sebkra}

$6160 \pm 150$

Ostrich egg shells from El Hadjar Sebkra, Oued Mya, Oasis area (30 52' N Lat, 5 ${ }^{\circ} 18^{\prime} \mathrm{E}$ Long), Algeria. Coll. 1970 and subm. by G. Aumassip. Comment: Neolithic culture (Aumassip, 1970). 
ALG-43. Hassi Chambi 3

Ostrich egg shells from Hassi Chambi, Oued Mya, Oasis area (30 $52^{\prime} \mathrm{N}$ Lat, 5 ${ }^{\circ} 18^{\prime} \mathrm{E}$ Long), Algeria. Coll. 1970 and subm. by G. Aumassip. Comment: Neolithic without pottery. Oldest age known for Neolithic samples in this part of Sahara (Aumassip, 1970).

\section{ALG-44. Adrar Tioueiine 4 \\ $5150 \pm 140$ 3200 B.c.}

Charcoal from Adrar Tioueiine, $35 \mathrm{~km} \mathrm{NW}$ of Silet Oasis area $\left(28^{\circ}\right.$ 48' N Lat, 4 16' E Long), Algeria. Coll. 1968 and subm. by G. Camps. Comment: Neolithic of Sudanese tradition with plentiful implements for grinding, stone bracelets, arrow points, and pottery.

$$
5270 \pm 140
$$

ALG-5. Hergla

3320 B.C.

Various terrestrial shells from Hergla, Gouvernorat de Sousse $\left(36^{\circ}\right.$ $1^{\prime} \mathrm{N}$ Lat, $10^{\circ} 28^{\prime} \mathrm{E}$ Long), Tunisia. Coll. 1970 and subm. by Harbi, Dept. de Préhistoire, Tunis. Comment: Neolithic culture.

\section{ALG-46. Rabah 19}

$7270 \pm 165$ 5320 B.c.

Fragments of ostrich egg shells from Rabah, Oued Djellal, Willaya Batna (34 $26^{\prime} \mathrm{N}$ Lat, $5^{\circ} 8^{\prime} \mathrm{E}$ Long), Algeria. Coll. 1970 and subm. by D. Grébenart. Comment: sample from level, 20 to $30 \mathrm{~cm}$, of Upper Capsian layer (Grébenart, 1971). This age follows our preceding date for this layer, ALG-17, -22, -23 (Grébenart, 1971).

\section{ALG-47. Chenoua (Rolland) \\ $13,330 \pm 280$ \\ 11,380 в.c. \\ Various terrestrial and sea shells, remains of food, from a cave,} Grotte Rolland, at Chenoua, near Tipasa, Willaya Algiers $\left(36^{\circ} 30^{\prime} \mathrm{N}\right.$ Lat, 2० 26' E Long), Algeria. Coll. 1970 and subm. by F. E. Roubet. Comment: sample of upper level of archaeologic layer. Ibero-Maurusian industry: Mouilien, Abris de la Mouilah.

\section{ALG-48. Tipasa 2}

$$
1870 \pm 100
$$

Charcoal from Matares, Tipasa, Willaya Algiers $\left(36^{\circ} 30^{\prime} \mathrm{N}\right.$ Lat, $2^{\circ} 26^{\prime}$ E Long), Algeria. Coll. 1969 and subm. by M. Bouchnaki. Comment: sample from Roman cupula tomb with an incineration urn, an oil lamp, and a Roman coin dated approx. from the end of 1st century to beginning of 2 nd.

\section{Grotte Capeletti series, Algeria}

Charcoal from Neolithic layer in a cave at Khanghet Si Mohamed Tahar, Bou Ahmar, Willaya Batna $\left(35^{\circ} 20^{\prime} 21^{\prime \prime} \mathrm{N}\right.$ Lat, $6^{\circ} 26^{\prime} 5^{\prime \prime} \mathrm{E}$ Long), Algeria. Coll. 1968 and subm. by Roubet. 
ALG-30. Grotte Capeletti 142

$4340 \pm 200$

Charcoal from upper 35 to $40 \mathrm{~cm}$ in Neolithic of Capsian tradition layer of cave.

\section{ALG-37. Grotte Capeletti 26}

$6530 \pm 250$ of cave.

Charcoal from basal Neolithic level, 314 to $317 \mathrm{~cm}$, 1st occupation

\section{ALG-50. Grotte Capeletti B3}

$4670 \pm 130$

Charcoal, 40 to $45 \mathrm{~cm}$ deep, in Neolithic of Capsian tradition layer.

ALG-51. Grotte Capeletti 143

$$
\mathbf{5 4 0} \pm \mathbf{9 0}
$$

Charcoal from uppermost platform in front of cave. Coll. with pottery of historic period, Berber manual trade.

General Comment: dwelling place of Neolithic of Capsian tradition searched for and excavation according to modern methods for marking and locating documents (Roubet, 1969).

\section{REFERENCES}

Aumassip, G., 1970, Notes sur le Néolithique Saharien: Liaison de l'assoc. Sénégalaise pour l'étude du quarternaire de l'ouest Africain (ASEQUA) Bull., Dakar. Fann. Sénégal, no. 25, Mar. 1970.

Brahimi, C., 1970, Encore des fouilles à Columnata: Rev. d'histoire et civilisation du Maghreb, Alger, Fac. des Lettres, in press.

Dedieu, B., 1965, Grotte du Djebel Zabaouine: Libyca, v. 13, Paris, Arts et Métiers Graphiques, p. 99-126.

Couvert, M., 1970, Etudes des charbons préhistoriques_Préparation des coupes minces et analyse des structures: C.A.R.A.P.E. travaux, Paris, Arts et Métiers Graphiques.

Grébenart, D., 1970, Problèmes du Néolithique près d'Ouled Djellal et de DjelfaBotma Si Mamar et Safiet Bou Rhenan: Libyca, v. 18, Paris, Arts et Mètiers Graphiques, p. 47-67.

— 1971, Un Gisement Capsien stratifié_Rabah près d'Ouled Djellal_Notes préliminaires: Libyca, v. 19, Paris, Arts et Métiers Graphiques, in press.

Rahmouni, O., Roussillot, C., and Armanet, F., 1970, Algiers radiocarbon measurements I: Radiocarbon, v. 12, p. 353-357.

1970, Laboratoire de datage par la methode du carbone-14 d'Alger: Libyca, v. 18, Paris, Arts et Métiers Graphiques, p. 9-22.

Roubet, C., 1968, Gisement du Dahmous el Ahmar: C.A.R.A.P.E. travaux, Paris, Arts et Métiers Graphiques.

1969, La Grotte Capeletti de Khanghet Si Mohamed Tahar (AurèsAlgérie)_Etudes préliminaires: Libyca, v. 17, Paris, Arts et Métiers Graphiques, p. 203-212.

Roubet, F. E., 1947, Contribution, à l'étude du peuplement préhistorique de l'Algérie Occidentale: Soc. géog. et archéol. de la prov. d'Oran Bull, Oran. 